\title{
ВЛИЯНИЕ ГРАВИТАЦИОННЫХ АНОМАЛИИ НА ЦИРКУЛЯЦИЮ ВОД МИРОВОГО ОКЕАНА
}

\section{1. УНДУЛЯЦИЯ ПОВЕРХНОСТИ ОКЕАНА}

Решение многих фундаментальных задач высшей геодезии и картографии осуществляется через знание строения Земли и распределения масс внутри ее. До семидесятых годов для изучения фигуры Земли использовали совместно с астрономическими методами и гравиметрический способ. Гравиметрическое определение фигуры Земли стало возможным лишь после накопления значительного количества сухопутных и морских измерений силы тяжести. Однако основным препятствием при определении геоида в пределах акваторий стала недостаточная изученность полей силы тяжести на океанах.

В начале семидесятых годов появился новый, более надежный и точный геометрический метод определения высот геоида на океанах, основанный на радиоальтиметрических наблюдениях со спутников, дающий, однако, прекрасные результаты лишь при определении низких гармоник в разложении гравитационного поля Земли. Детальное же изучение гравитационного поля Земли остается задачей традиционной гравиметрии. Низкие гармоники обусловлены региональными аномалиями силы тяжести, вызванными крупными аномальными телами глубокого заложения.

На основе альтиметрических данных составлена карта высот геоида над эллипсоидом вращения (рис. 1). Глубины залегания возмущающих масс оцениваются по совместным картам высот геоида и аномалий силы тяжести (Грушинский, 1983; Тараканов, 1982) (табл. 1).

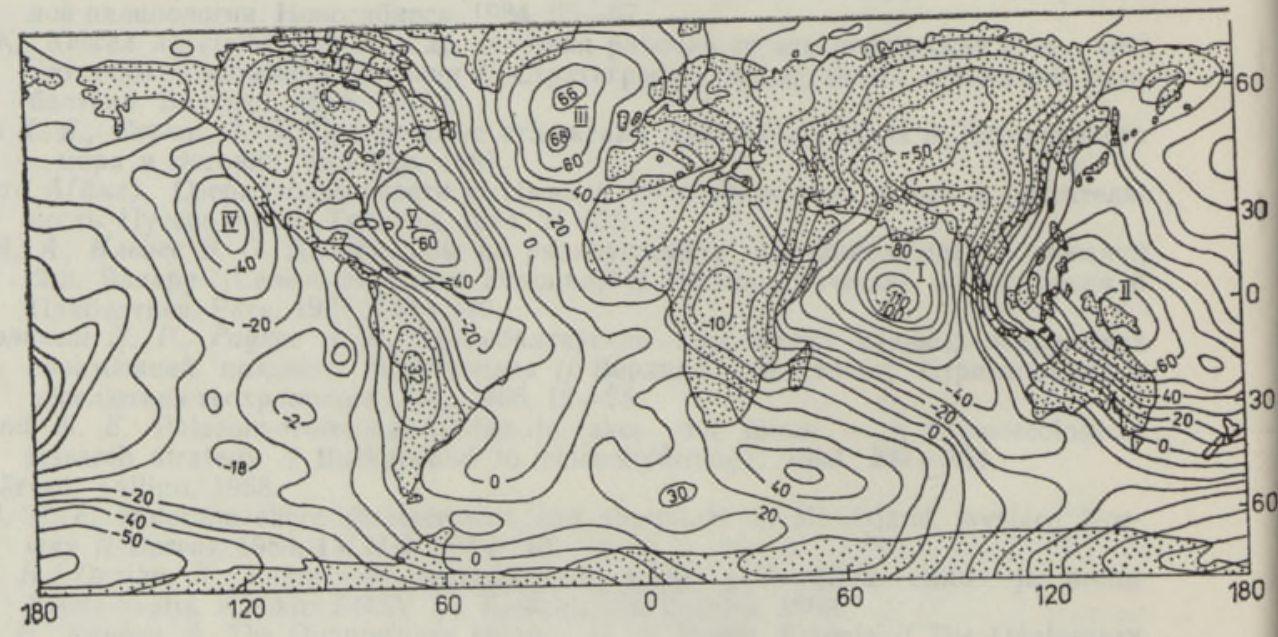

Рис. 1. Крупнейшие аномалии геоида (по Тараканову, 1982): I - Индийская, II Австралийская, III - Северо-Атлантическая, IV - Калифорнийская, V - Карнбская. 
Глубина залегания масс, вызвавших крупнейшие аномалии геоида

\begin{tabular}{r|l|c|c|c}
\hline $\begin{array}{c}\text { № } \\
\text { на } \\
\text { рис. 1 }\end{array}$ & \multicolumn{1}{|c|}{ Аномалия } & $\begin{array}{c}\text { Величнна } \\
\text { аномалии, } \\
\text { мГал }\end{array}$ & $\begin{array}{c}\text { Высота } \\
\text { геонда, } \\
\text { м }\end{array}$ & $\begin{array}{c}\text { Глубина залегания } \\
\text { возмущающих масс, } \\
\text { вызвавших анома- } \\
\text { лию, км }\end{array}$ \\
\hline I & Индийская & -50 & -110 & 840 \\
II & Австралийская & +30 & +70 & 960 \\
III & Северо-Атлантическая & +30 & +68 & 1000 \\
IV & Калифорнийская & -30 & -50 & 770 \\
V & Карибская & -40 & -60 & 630
\end{tabular}

Целью настоящей статьи является разработка расчетной схемы для вычисления ундуляции поверхности океана в районе гравитационной аномалии, а также горизонтального компонента и горизонтального градиента вертикального компонента аномальной притягивающей силы. В дальнейшем результаты этой статьи используются при оценке скорости движения океанических вод, вызванных крупными аномалиями силы тяжести.

При статическом равновесии сил поверхность океана можно рассматривать как соответствующий геоид, т. е. высота геоида и есть ундуляция поверхности океана. Возникновение ундуляции поверхности океана в районе гравитационной аномалии можно объяснить при помощи следующего примера. Если в океан упадет массивный метеорит, в этом месте за счет увеличения ускорения силы тяжести возникнет положительная гравитационная аномалия.

Предположим, что перед падением метеорита в воде существует статическое равновесие сил. Масса метеорита начнет притягивать воду, в результате чего возникнет дополнительное давление с нарушением состояния равновесия в воде

$$
d p=-0 g_{r} d r,
$$

где $d p-$ дополнительное давление в элементарном слое из-за притяжения воды метеоритом; $Q$ - плотность воды; $r$ - радиус элементарного сферического слоя; $d r$ - толщина элементарного слоя; $g_{r}-$ напряженность поля притяжения метеорита (знак «минус» означает, что радиус измеряется в обратном направлении относительно давления).

Теперь $g_{r}$ имеет вид

$$
g_{r}=-\frac{G M}{r^{2}},
$$

где $G$ - гравитационная постоянная; $M$-масса метеорита; $r$-радиусвектор от центра массы метеорита до центра исследуемого элементар-
ного слоя. Дополнительное давление слоя воды $r_{0}-r$ на поверхности метеорита

$$
\Delta p=\int_{r_{0}}^{r} \varrho g_{r} d r
$$

где $r_{0}-$ радиус метеорита.

Если $\varrho=$ конст., тогда (3) можно записать в виде

$$
\Delta p=\varrho \int_{r_{\varrho}}^{r} g_{r} d r=\varrho G M \int_{r_{\varrho}}^{r} \frac{d r}{r^{2}}=\varrho G M\left(\frac{1}{r_{0}}-\frac{1}{r}\right) .
$$




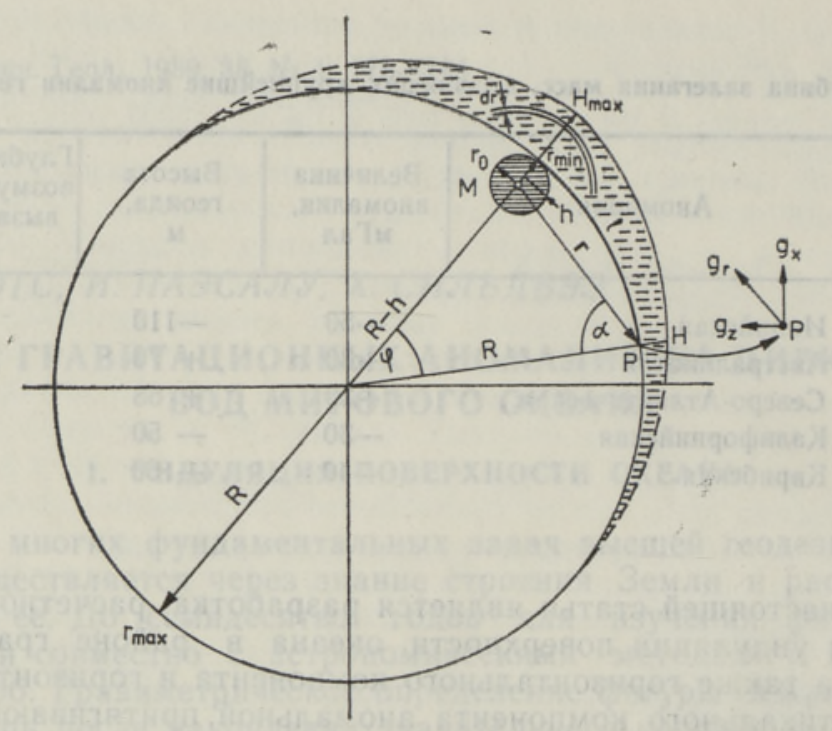

Рис. 2. Принципиальная схема ундуляции поверхности океана над возмущающей массой.

Максимальное дополнительное давление будет достигнуто, если $r=r_{\max }$ и минимальное, если $r=r_{\min }$ (рис. 2).

Имея в виду, что $G M \cdot r^{-1}$ представляет собой потенциал $(V)$ гравитационного поля метеорита в точке с радиусом-вектором $r$, формулу (4) можно переписать в виде

$$
\Delta p=\varrho\left(V_{0}-V\right) .
$$

Как видно, дополнительное давление слоя воды определено разностью гравитационных потенциалов метеорита на краях этого слоя. Тогда дополнительное давление всего слоя воды на поверхности метеорита будет

$$
\Delta p=\varrho\left(V_{0}-V_{\min }\right) .
$$

Вследствие дополнительного давления, действующего со сторон, и согласно закону Паскаля, уровень воды над метеоритом будет повышаться. Гравитационное поле Земли препятствует этому повышению. Вследствие этого уровень воды поднимается только на величину $\Delta H$, определяемую из формулы

$$
\begin{gathered}
\Delta H_{i} \gamma=\frac{1}{\varrho} \Delta p_{i}, \\
\Delta H_{i}=\frac{1}{\gamma \cdot \varrho} \cdot \Delta p_{i},
\end{gathered}
$$

где $\Delta p_{i}$ - дополнительное давление в точке $i$, определяемое через $\left(V_{i}-V_{\min }\right)$ (рис. 2$) ; \gamma-$ напряженность нормального поля силы тяжести Земли; $V$ - гравитационный потенциал метеорита. Теперь формулу (7) можно переписать в виде

$$
\Delta H_{i}=\frac{V_{i}-V_{\min }}{\gamma} .
$$

Для определения точек на физической поверхности океана введем координату $H$, измеряемую от сфероида по нормали (рис. 2). 
Максимальная высота подъема уровня воды $\left(H_{\max }\right)$ находится в пункте, где $r=r_{\min }$ или $V_{l}=V_{\max }$

$$
H_{\max }=\frac{V_{\max }-V_{\min }}{\gamma} ; \quad V_{\max }=\frac{G M}{r_{\min }} ; \quad V_{\min }=\frac{G M}{r_{\max }} .
$$

Для определения высоты $(H)$ всех пунктов применяется формула

или

$$
H=\frac{V-V_{\min }}{\gamma} ; \quad V=\frac{G M}{r}
$$

$$
H=\frac{G M}{\gamma}\left(\frac{1}{r}-\frac{1}{r_{\max }}\right)
$$

где $r_{\max }=2 R-h ; R-$ средний радиус Земли; $h-$ глубина возмущающей массы (метеорита) от поверхности сфероида.

С целью иллюстрации полученных результатов, предположим, что в океан на глубину 2000 м пал железный шарообразный метеорит плотностью $\varrho=7900 \mathrm{Kг} / \mathrm{M}^{3}$ и радиусом $1000 \mathrm{~m}$.

В таком случае масса метеорита составляет $M=3,31 \cdot 10^{13} \mathrm{Kr}$.

Теоретически масса метеорита должна оказывать влияние на воды океана в пределах всего Земного шара, практически же изменение уровня воды заметно только вблизи места падения метеорита.

$$
\begin{aligned}
& r_{1}=r_{\min }=2 \mathrm{KM} \\
& r_{2}=5 \mathrm{KM} \\
& r_{3}=25 \mathrm{KM} \\
& r_{4}=50 \mathrm{KM} \\
& r_{5}=100 \mathrm{KM} \\
& r_{6}=r_{\max }=12742-2=12740 \mathrm{KM}
\end{aligned}
$$

$$
\begin{aligned}
& H_{1}=H_{\max }=11,25 \mathrm{~cm} \\
& H_{2}=4,50 \mathrm{~cm} \\
& H_{3}=0,90 \mathrm{~cm} \\
& H_{4}=0,45 \mathrm{~cm} \\
& H_{5}=0,22 \mathrm{~cm} \\
& H_{6}=0=H_{\min }
\end{aligned}
$$

Из приведенного примера видно, что достаточно учитывать влияние притяжения метеорита в радиусе 100 км. При дальнейшем увеличении расстояния подъем воды составляет менее 2 мм.

В предложенной схеме расчета высо́ты $H$ даны по отношению к поверхности сфероида. Если считать поверхностью относимости поверхность референц-эллипсоида, то $H$ различаются, но не более, чем на $\Delta= \pm H \cdot 10^{-4} \mathrm{~m}$, т. е. порядка $0,1 \mathrm{мм}$.

При расчете высоты $H$ относительно референц-эллипсоида 1967 г. нужно во всех формулах средний радиус $R$ заменить радиус-вектором $R_{p}$

$$
R_{p}=\sqrt{\frac{a^{2} \cos ^{2} B+a^{2}\left(1-e^{2}\right)^{2} \sin ^{2} B}{1-e^{2} \sin ^{2} B}},
$$

где $a$ - большая полуось, 6378160 м; $b$ - малая полуось, 6356774 м; $B-$ географическая широта исследуемого пункта; $e^{2}=1-b^{2} / a^{2}=$ $=6,6948 \cdot 10^{-3}$.

Вызванную метеоритом ундуляцию поверхности океана мы рассчитали как функцию расстояния $r$ от центра метеорита до исследуемой точки. Ундуляцию поверхности океана на расстоянии $l$, измеренном по поверхности сфероида от центра гравитационной аномалии, можно рассчитать при помощи угла $\varphi$ между центром аномалии $M$ и исследуемым пунктом $P$ (по рис. 2)

$$
\varphi=\frac{l}{R},
$$

где $R$ - средний радиус Земли. 


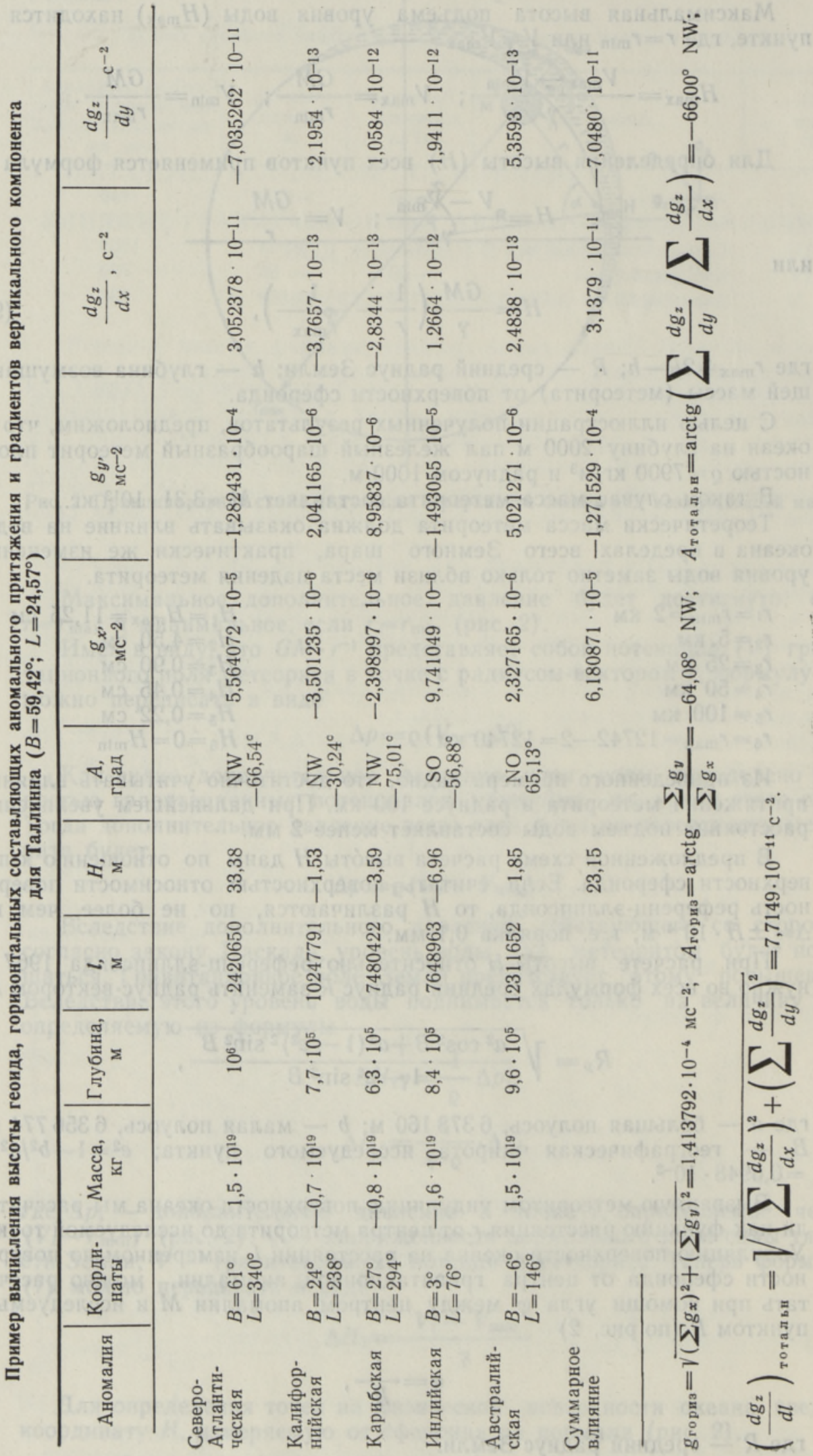




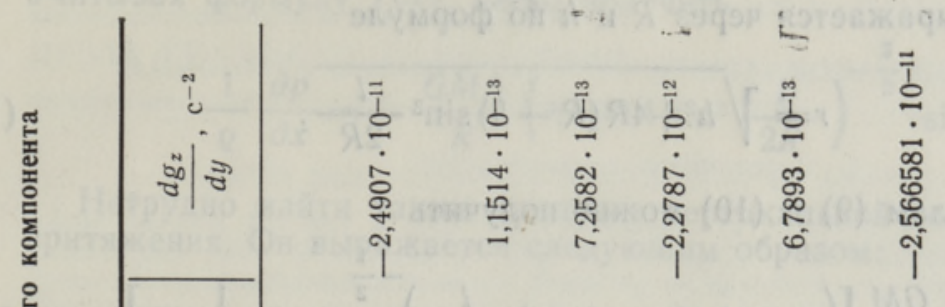

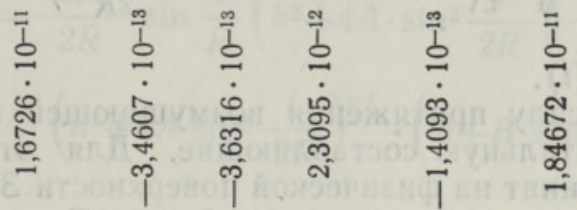

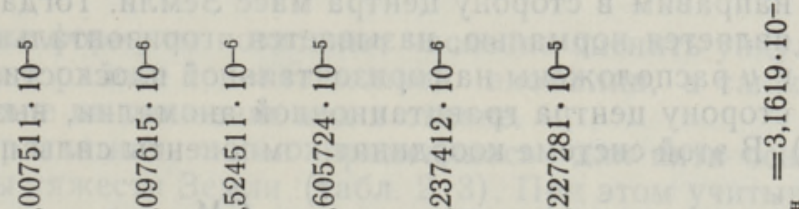

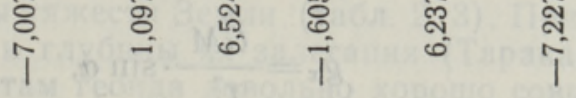

! 의 의

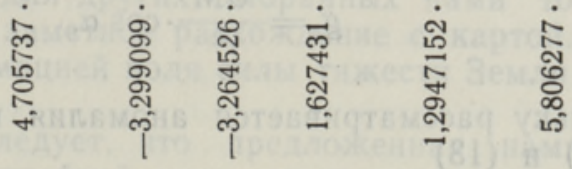

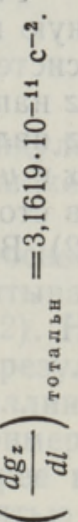

$\times$ II

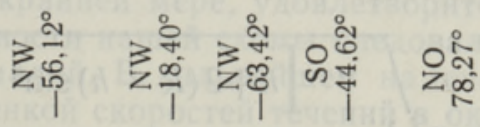

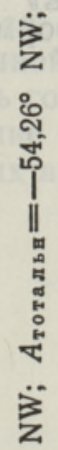

递要

ช⿳ㅠํ

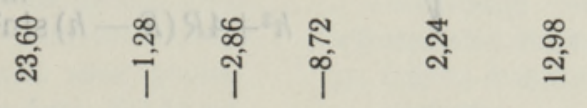

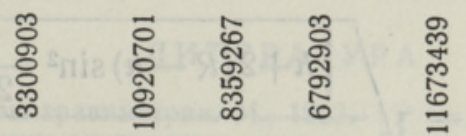

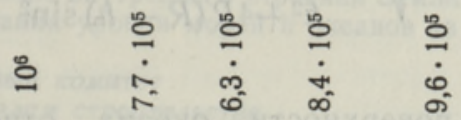

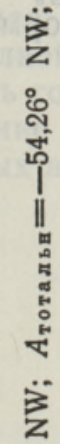

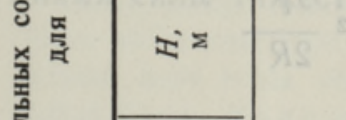

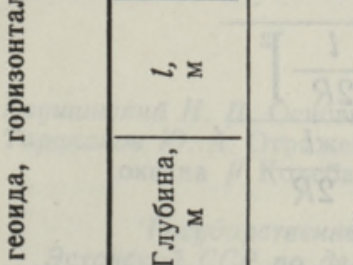

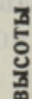

苂

$\div \quad \div \quad \div \quad$

$\stackrel{\dot{0}}{i} i^{\infty} \dot{i} \stackrel{\dot{j}}{\dot{0}}$

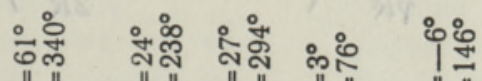

III II II

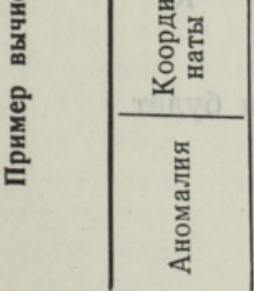

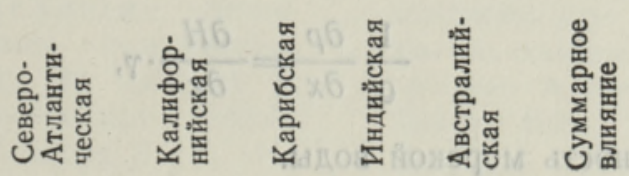

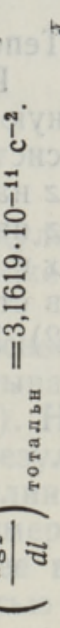

สิt

ì

空 
Тогда $r$ выражается через $R$ и $h$ по формуле

$$
r=\sqrt{h^{2}+4 R(R-h) \sin ^{2} \frac{l}{2 R}} .
$$

По формулам (9) и (10) можно получить

$$
H=\frac{G M}{\gamma}\left[\left(h^{2}+4 R(R-h) \sin ^{2} \frac{l}{2 R}\right)^{-\frac{1}{2}}-\frac{1}{2 R-h}\right] .
$$

Теперь $H=H(l)$.

Разделим силу притяжения возмущающей массы $M$ на вертикальную и горизонтальную составляющие. Для этого подберем местную систему координат на физической поверхности Земли. Вертикальную ось $z$ направим в сторону центра масс Земли. Тогда плоскость, для которой $z$ является нормалью, называется горизонтальной. Координатные оси $x$ и $y$ расположены на горизонтальной плоскости, при этом $x$ направлена в сторону центра гравитационной аномалии, вызванной массой $M$ (рис. 2). В этой системе координат компоненты силы притяжения выражаются

$$
\begin{aligned}
& g_{x}=\frac{G M}{r^{2}} \cdot \sin \alpha, \\
& g_{z}=\frac{G M}{r^{2}} \cdot \cos \alpha,
\end{aligned}
$$

( $g_{y}=0$, поскольку рассматривается аномалия круговой симметрии). В формулах (12) и (13)

$$
\begin{gathered}
\sin \alpha=\sqrt{1-\frac{\left[h+2(R-h) \sin ^{2} \frac{l}{2 R}\right]^{2}}{h^{2}+4 R(R-h) \sin ^{2} \frac{l}{2 R}}}, \\
\cos \alpha=\sqrt{\frac{\left[h+2(R-h) \sin ^{2} \frac{l}{2 R}\right]^{2}}{h^{2}+4 R(R-h) \sin ^{2} \frac{l}{2 R}}} .
\end{gathered}
$$

Найдем наклон поверхности океана относительно касательной поверхности сфероида. Продифференцировав формулу (11) по $x$, получим

$$
\frac{\partial H}{\partial x}=-\frac{G M A}{\gamma R}\left(h^{2}+4 A \sin ^{2} \frac{l}{2 R}\right)^{-\frac{3}{2}} \cdot \sin \frac{l}{R},
$$

где $A=R(R-h)$.

Градиент давления в горизонтальном направлении будет

$$
\frac{1}{\varrho} \frac{\partial p}{\partial x}=\frac{\partial H}{\partial x} \cdot \gamma
$$

где $\varrho-$ плотность морской воды. 
Учитывая формулу (14), легко получить

$$
\frac{1}{\varrho} \frac{\partial p}{\partial x}=-\frac{G M A}{R}\left(h^{2}+4 A \sin ^{2} \frac{l}{2 R}\right)^{-\frac{3}{2}} \cdot \sin \frac{l}{R}
$$

Нетрудно найти также градиент вертикальной составляющей силы притяжения. Он выражается следующим образом:

$$
\begin{gathered}
\frac{\partial g_{z}}{\partial x}=\frac{G M}{2 R} \cdot \sin \frac{l}{R}\left(h^{2}+4 A \cdot \sin ^{2} \frac{l}{2 R}\right)^{-\frac{3}{2}} \times \\
\times\left[B-6 A \cdot\left(h^{2}+4 A \sin ^{2} \frac{l}{2 R}\right)^{-1} \cdot\left(h+B \cdot \sin ^{2} \frac{l}{2 R}\right)\right]
\end{gathered}
$$

где $B=2(R-h)$.

Выведенные выше формулы позволяют численно оценить ундуляцию поверхности океана в районе гравитационной аномалии, а также возможное ее воздействие на движение вод в океане.

Разработанная расчетная схема применялась для пяти основных аномалий поля силы тяжести Земли (табл. 2,3 ). При этом учитывались аномальные массы и глубины их залегания (Тараканова, 1982). Наши результаты по высотам геоида довольно хорошо совпадают с результатами, приведенными на рис. 1 для исследованных точек Таллинна и Москвы. Но расчет для других выбранных нами точек, например для Парижа, дает уже заметное расхождение с картой. Это скорее всего связано с аппроксимацией поля силы тяжести Земли только пятью аномалиями.

Из сказанного следует, что предложенная нами расчетная схема функционирует, по крайней мере, удовлетворительно. Для полной проверки работоспособности нашей схемы следовало бы учитывать гораздо большее число аномалий. В дальнейшем намечается выполнение такой работы вместе с оценкой скоростей течений в океане, вызванных аномалиями силы тяжести.

\section{ЛИ Т Е Р А Т У Р А}

Грушинский Н. П. Основы гравиметрин. М., 1983.

Тараканов Ю.А. Отражение внутреннего строения Земли в рельефе водной поверхности океана // Колебания уровня морей и океанов за 15000 лет. М., 1982, 23-51.

Государственный комитет

Эстонской ССР по делам строительства

Институт геологии

Академии наук Эстонской ССР
Поступила в редакцию $8 /$ VI 1988 


\section{GRAVITATSIOONIANOMAALIATE MOJU VEE TSIRKULATSIOONILE MAAILMAOOKEANIS}

\section{Ookeanipinna undulatsioonid}

Analüütilisel kujul on tuletatud arvutusskeem ookeanipinna (geoidi) kõrguse hindamiseks anomaalsete masside kohal, kusjuures on lähtutud anomaalse massi külgetômbejõust tingitud lisarôhust vees. Valemid on tuletatud ka anomaalse külgetõmbejỗu vertikaalkomponendi horisontaalgradientide arvutamiseks. Viimaseid vajatakse edaspidi hindamaks gravitatsioonianomaaliate mõju vee liikumise kiirusele. Năited on toodud Tallinna ja Moskva punkti kohta.

\section{T. VALLAOTS, J. PAESALU, H. SILDVEE}

\section{GRAVITY ANOMALIES EFFECT ON THE WATER CIRCULATION IN THE WORLD OCEAN}

\section{Ocean surface undulations}

A procedure has been worked out to estimate the ocean surface (geoid) undulations arising from the Earth's anomalous masses. We proceed from the concept of additional pressure, caused by the anomalous mass gravitational attraction. The formulas are derived not only for the calculation of geoid height but for the horizontal gradients of anomalous gravity vertical component as well. Some numerical examples are presented (Tables 2 and 3 ). 